\title{
A Influência do Acesso à Saúde na Apresentação Clínica das Neoplasias Benignas e Malignas da Tireoide em Pacientes Submetidos à Tireoidectomia Total
}

\author{
The Influence of Access to Health in The Clinical Presentation of Benign and \\ Malignant Thyroid Neoplasms in Patients Undergoing Total Thyroidectomy
}

\section{RESUMO}

Objetivo: Avaliar a influência do acesso à saúde na apresentação clínica e histopatológica das neoplasias tireoidianas benignas e malignas em pacientes submetidos à tireoidectomia total. Metodologia: Foi realizada a revisão de prontuários de pacientes submetidos à tireoidectomia total em um serviço de cirurgia de cabeça e pescoço do município de Guarapuava, Paraná, Brasil, entre os anos de 2003 e 2017. Foram coletadas informações demográficas (idade, sexo, estado civil e tipo de acesso à saúde) e clínicas (volume total da tireoide, diagnóstico patológico, peso dos espécimes pós-cirúrgicos e estadiamento patológico e clínico). A amostra foi dividida em dois grupos, pacientes atendidos pelo SUS e saúde suplementar. Para a análise dos dados foi utilizando o software estatístico SPSS versão 20.0. O nível de significância foi $p<0,05$. Resultados: Dentre os 315 pacientes estudados, $104(33,02 \%)$ foram tratados pelo SUS e $211(66,98 \%)$ pela saúde suplementar. Nos dois grupos, o volume total da tireoide foi menor em pacientes com menos de 45 anos, nas mulheres e nas neoplasias malignas ( $p<0,001)$, enquanto, o peso do espécime cirúrgico foi menor em pacientes com menos de 45 anos, em pacientes atendidos pela saúde suplementar $(p<0,001)$ e nas neoplasias malignas $(p<0,049)$. Os casos atendidos pelo SUS apresentaram maior volume total da glândula $(p<0,001)$ e maior peso do espécime cirúrgico $(p<0,001)$, além de maior incidência de metástases regionais $(p=0,030)$. Conclusão: $O$ tipo de acesso à saúde influencia na apresentação clínica das neoplasias da tireoide em relação ao volume total da tireoide, presença de metástases regionais e peso do espécime cirúrgico.

\section{DESCRITORES}

Acesso à saúde. Tireoide. Neoplasias da Tireoide. Cirurgia da Tireoide.

\begin{abstract}
Objective: To evaluate the influence of access to health care on the clinical and histopathological presentation of benign and malignant thyroid neoplasms in patients undergoing total thyroidectomy. Methodology: A review of medical records of patients undergoing total thyroidectomy was performed in a head and neck surgery service in the city of Guarapuava, Paraná, Brazil between 2003 and 2017. Demographic (age, sex, marital status and type of access to health) and clinical (total thyroid volume, pathological diagnosis, weight of postoperative specimens, and pathological and clinical staging) information were collected. The sample was divided into two groups, patients treated by public health insurance (SUS) and private health insurance. For data analysis, the statistical software SPSS version 20.0 was used. The significance level was $p<0.05$. Results: Among the 315 patients studied, 104 (33.02\%) were public patient (SUS) and 211 (66.98\%) had private insurance coverage. In both groups, the total thyroid volume was lower in patients under 45 years of age, in women and malignant neoplasms $(p<0.001)$. The weight of the surgical specimen was lower in patients under 45 years of age, in patients with private insurance coverage $(p<0.001)$, and in malignant neoplasms $(p<0.049)$. The cases of public patients had higher total gland volume $(p<0.001)$ and higher weight of the surgical specimen $(p<0.001)$, in addition to a higher incidence of regional metastases $(p=0.030)$. Conclusion: The type of access to health care influences the clinical presentation of thyroid neoplasms in relation to the total volume of the thyroid, presence of regional metastases and weight of the surgical specimen.
\end{abstract}

\section{DESCRIPTORS}

Health care access. Thyroid. Thyroid neoplasms. Thyroid surgery.

\footnotetext{
${ }^{1}$ Fisioterapeuta. Mestre pela Universidade Estadual do Centro-Oeste (UNICENTRO), Guarapuava, Paraná, Brasil.

${ }^{2}$ Acadêmico de Medicina da Pontifícia Universidade Católica do Paraná (PUC), Curitiba, Paraná, Brasil.

${ }^{3}$ Doutor, Professor Adjunto da Universidade Estadual do Centro-Oeste (UNICENTRO), Guarapuava, Paraná, Brasil.
} 
$A^{5}$ s desigualdades presentes em nossa sociedade ocasionam um evidente desequilíbrio no acesso à saúde ${ }^{1}$. Entendese por desigualdade em saúde a diferença no acesso a recursos e a fatores que influenciam a saúde e que são dependentes de circunstâncias e contextos sociais, especialmente de políticas públicas ${ }^{2,3}$.

O processo de saúde e doença deve ser pensado do ponto de vista multifatorial, considerando os diversos fatores de risco sociais, dentre eles, o estilo de vida, e condições socioeconômicas, que impactam no acesso à saúde e no enfrentamento de diversas doenças ${ }^{4}$, dentre elas as doenças crônicas não transmissíveis ${ }^{1,5}$, influenciando a sua incidência e apresentação. Nos casos de câncer, por exemplo, estudos evidenciam o impacto de precárias condições socioeconômicas na incidência bem como diagnóstico tardio, além de maior dificuldade de tratamento adequado. Estes fatores impactam o prognóstico, com menor sobrevida e maior risco de óbito pela doença, incluindo alguns tipos de câncer potencialmente curáveis ${ }^{1,5-7}$

Neoplasias tireoidianas, benignas e malignas, manifestam-se geralmente pela presença de nódulos tireoidianos. Estudos epidemiológicos relatam que $4 \%$ a $7 \%$ das mulheres e $1 \%$ dos homens adultos apresentam nódulo palpável ${ }^{8}$. Achados de ultrassom revelam prevalência maior, chegando a mais de $60 \%$, com maior incidência em mulheres e idosos ${ }^{8,9}$. Apesar de a grande maioria representar lesões benignas, é necessário excluir o câncer da tireoide, que ocorre em $5 \%$ a $10 \%$ dos casos $^{10,11}$.

Fatores sociais relacionados à saúde também podem ser observados nas neoplasias tireoidianas. Nos casos de bócio, uma neoplasia benigna da tireoide ainda altamente prevalente em diferentes regiões do mundo e considerada um problema de saúde pública cuja principal causa é a deficiência de iodo, sabe-se que países mais pobres da África estão entre os que mais apresentam carência de iodo ${ }^{12}$, elevando a prevalência total de bócio na população, em geral a $28,3 \%$, que é considerada a maior taxa entre os continentes ${ }^{13}$.

Em relação às neoplasias malignas, além de serem as mais frequentes do sistema endócrino, existem relatos de discrepância na apresentação clínica da doença associada ao estado socioeconômico, incluindo tamanho do tumor e a presença de metástases ${ }^{14-16}$.

No presente estudo foi avaliada a influência do acesso à saúde na apresentação clínica e histopatológica das neoplasias tireoidianas benignas e malignas no momento do tratamento em pacientes submetidos à tireoidectomia total na Cidade de Guarapuava, Paraná, Brasil, que possui uma estimativa populacional de 181.504 habitantes, com densidade demográfica de 57,1 hab./ $\mathrm{km}^{217}$.

\section{METODOLOGIA}

Trata-se de um estudo analítico observacional transversal ${ }^{18}$, sendo realizada a revisão de prontuários de um serviço de cirurgia de cabeça e pescoço do Município de Guarapuava, Estado do Paraná, Brasil, entre os anos de 2003 e 2017. O presente estudo foi aprovado pelo Comitê de Ética em Pesquisa da Universidade Estadual do Centro-Oeste, sob o Parecer $n^{\circ}$. 2.278.774. 
População de estudo

Foram incluídos neste estudo pacientes com diagnóstico de neoplasia tireoidiana benigna ou maligna submetidos à tireoidectomia total eletiva, entre os anos de 2003 e 2017. Foram excluídos pacientes submetidos à tireoidectomia parcial e prontuários com dados insuficientes ou incompletos.

Os dados foram selecionados por meio dos registros cirúrgicos. A partir dessas informações, foi realizada a triagem dos prontuários pelo registro médico eletrônico. $\mathrm{A}$ amostra foi dividida em dois grupos, baseados no tipo de acesso à saúde: saúde pública (SUS) ou saúde suplementar (planos e seguros de saúde privados). Foram analisadas as informações demográficas, incluindo a idade, o sexo, o estado civil e o tipo de acesso à saúde. As variáveis clínicas coletadas foram o volume total da tireoide estimado pela ultrassonografia, o tipo de doença da tireoide, o diagnóstico patológico, o peso dos espécimes pós-cirúrgicos e o estadiamento patológico TNM (T: tumor primário; $\mathrm{N}$ : linfonodos: $\mathrm{M}$ : metástases regionais) e clínico nos casos de neoplasias.

O volume total da tireoide foi obtido pelo exame de ultrassom realizado no período pré-operatório. Nos casos de prontuários sem essa informação o volume foi calculado pela soma dos volumes dos lobos direito e esquerdo, seguindo o padrão estabelecido para medir o volume de estruturas elípticas pela fórmula ${ }^{19,20}$ :

dimensão anteroposterior $\mathrm{x}$

dimensão mediolateral $x$ dimensão craniocaudal $x$ fator de correção $(\pi / 6)$.
O istmo foi excluído do cálculo do volume total da tireoide, de acordo com o método comum validado por estudos anteriores ${ }^{20,21}$

O diagnóstico patológico foi dividido em doença benigna e doença maligna. Foram consideradas benignas, a hiperplasia nodular, os adenomas, cisto benigno ou nódulo coloide, bócio coloide, bócio multinodular, tireoidite de Hashimoto, tireoidite linfocítica e doença de Graves. Nos casos de doenças malignas foram contemplados os carcinomas papilífero, folicular, medular, anaplásico e de células de Hurthle. Nesses últimos casos, o estadiamento TNM foi realizado de acordo com as recomendações da American Joint Committee on Cancer ${ }^{22}$.

O peso dos espécimes cirúrgicos, o diagnóstico patológico e o estadiamento patológico foram obtidos a partir dos laudos anatomopatológicos.

Análise dos dados

Para análise dos dados foi utilizando o software estatístico SPSS versão 20.0. Inicialmente testaram-se os pressupostos analíticos, normalidade e homogeneidade das variâncias, aplicando-se os testes de Shapiro-Wilk e Kolmogorov-Smirnov. Violado os pressupostos, os dados foram analisados a partir de técnicas não paramétricas, utilizando o teste do Qui-quadrado para as comparações entre o SUS e o privado e os testes de Mann-Whitney e Kruskal-Wallis para as comparações das variáveis demográficas e clínicas com o volume total da glândula e com o volume e peso do espécime cirúrgico. $\mathrm{O}$ nível de significância considerado foi $p<0,05$. 


\section{RESULTADOS}

Inicialmente foram triados para a pesquisa, pelas informações contidas nos registros cirúrgicos, 487 pacientes, sendo que a amostra final foi composta por 315 indivíduos. Dentre estes, 104 pacientes realizaram a cirurgia pelo SUS e 211 pela saúde suplementar. As informações do processo de seleção encontram-se na Figura 1.

A idade média dos pacientes estudados, no momento da cirurgia, foi de $47,1 \pm 14,66$ anos, sendo $46,77 \pm 8,48$ anos para as mulheres e 48,92 $\pm 5,65$ anos para os homens. Na maior parte da amostra os pacientes apresentavam idade acima de 45 anos $(56,50 \%)$, eram mulheres $(87,61 \%)$, realizaram a cirurgia pela saúde suplementar
(66,98\%) e possuíam cônjuge $(71,42 \%)$. A maioria não possuía presença concomitante de outra doença da tireoide $(85,71 \%)$, sendo que, dos que apresentaram alguma, as mais prevalentes foram a tireoidite de Hashimoto $(6,98 \%)$ e tireoidite linfocítica $(6,98 \%)$. A mediana do volume total da glândula estimado pela US foi de $29,4 \mathrm{~cm}^{3}$ (IC: 15,10 - 55,45), enquanto a mediana do peso do espécime cirúrgico foi de 30 gramas (IC: 20 - 70). Em relação ao diagnóstico patológico $68,58 \%(n=216)$ apresentava doença benigna, enquanto $31,42 \%(n=99)$ possuía alguma doença maligna da tireoide.

As comparações entre as características clínicas e demográficas entre os grupos, baseados no tipo de acesso à saúde pelo qual realizaram a cirurgia (SUS ou saúde suplementar), são apresentadas na Tabela 1.

Figura 1. Seleção dos pacientes

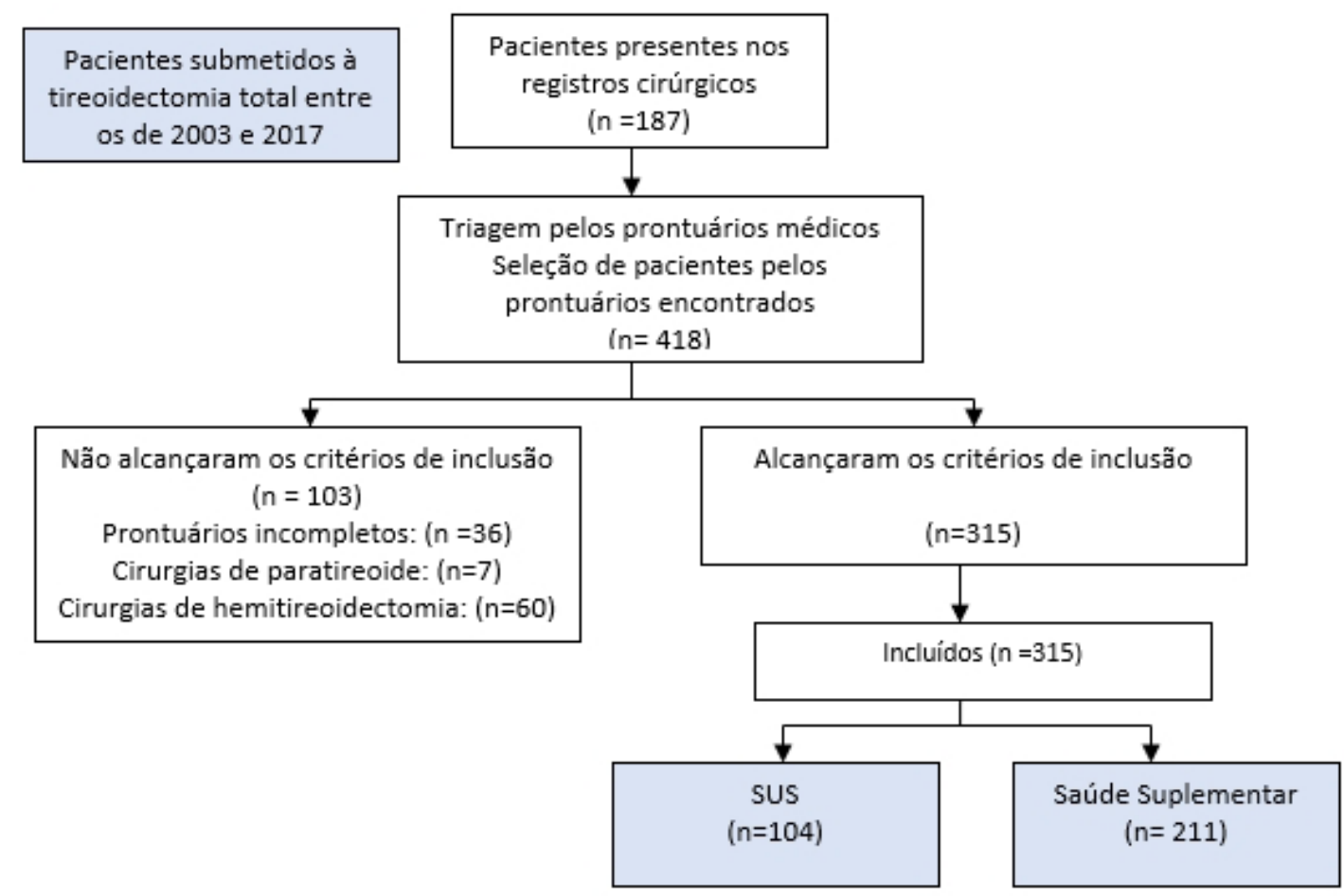


Tabela 1. Características demográficas e clínicas dos pacientes, comparação SUS e saúde suplementar

\begin{tabular}{|c|c|c|c|}
\hline \multirow[t]{2}{*}{$\begin{array}{c}\text { Caraterísticas } \\
\text { Demográficas e Clínicas }\end{array}$} & $\begin{array}{c}\text { SUS } \\
\mathrm{n}=104 \\
\mathrm{n}(\%)\end{array}$ & $\begin{array}{c}\text { Saúde Suplementar } \\
n=211 \\
n(\%) \\
\end{array}$ & \multirow{3}{*}{$\begin{array}{c}\mathrm{p} \\
0,624 \\
\end{array}$} \\
\hline & \multicolumn{2}{|c|}{$\mathrm{n}=315$} & \\
\hline Idade na cirurgia & $47,68( \pm 14,45)$ & $46,81( \pm 14,79)$ & \\
\hline $\begin{array}{l}\text { Maior idade } \\
<45 \\
\geq 45\end{array}$ & $\begin{array}{l}46(44,2) \\
58(55,8)\end{array}$ & $\begin{array}{c}91(43,1) \\
120(56,9)\end{array}$ & 0,904 \\
\hline $\begin{array}{l}\text { Sexo } \\
\text { Masculino } \\
\text { Feminino }\end{array}$ & $\begin{array}{l}15(14,4) \\
89(85,6)\end{array}$ & $\begin{array}{l}24(11,4) \\
187(88,6)\end{array}$ & 0,469 \\
\hline $\begin{array}{l}\text { Estado civil } \\
\text { Com companheiro } \\
\text { Sem companheiro }\end{array}$ & $\begin{array}{l}74(71,2) \\
30(28,8)\end{array}$ & $\begin{array}{l}151(71,6) \\
60(28,4)\end{array}$ & 0,940 \\
\hline $\begin{array}{l}\text { Presença concomitante de } \\
\text { doença tireoidiana } \\
\text { Nenhuma } \\
\text { Doença de Graves } \\
\text { Tireoidite de Hashimoto } \\
\text { Tireoidite Linfocítica } \\
\end{array}$ & $\begin{array}{c}88(84,6) \\
- \\
7(6,7) \\
9(8,7) \\
\end{array}$ & $\begin{array}{c}182(86,3) \\
1(0,5) \\
15(7,1) \\
13(6,2) \\
\end{array}$ & 0,820 \\
\hline Volume total da tireoide & $46,6(25,5-115,4)$ & $24,7(14,6-46,8)$ & $<0,001$ \\
\hline $\begin{array}{l}\text { Diagnóstico Patológico }{ }^{\mathrm{a}} \\
\text { Benigno } \\
\text { Maligno }\end{array}$ & $\begin{array}{l}70(67,3) \\
34(32,7) \\
\end{array}$ & $\begin{array}{l}146(69,2) \\
65(30,8) \\
\end{array}$ & 0,797 \\
\hline $\begin{array}{l}\text { Biópsia } \\
\text { Peso do espécime cirúrgico }\end{array}$ & $40(25,0-130,0)^{\mathrm{b}}$ & $25,5(15,0-60,0)^{c}$ & $<0,001$ \\
\hline
\end{tabular}

As comparações mostraram-se significativas para as variáveis de volume total da glândula e peso do espécime cirúrgico. Quanto às variáveis, idade, sexo, estado civil, presença concomitante de doença da tireoide e diagnóstico patológico não apresentaram diferenças significativas.

Em relação aos casos com diagnóstico de neoplasia maligna, a única diferença encontrada foi na presença de metástases regionais $(p=0,03)$ (Tabela 2$)$.

O volume da glândula mostrou associação significativa com a idade, o sexo, o tipo de acesso à saúde, a presença concomitante de doença tireoidiana e o diagnóstico patológico (Tabela 3).

A Tabela 4 apresenta a associação entre o peso do espécime cirúrgico e as variáveis demográficas e clínicas. Foi observada associação com a idade, o tipo de acesso à saúde, a presença concomitante de doença tireoidiana e o diagnóstico patológico.

\section{DISCUSSÃO}

As desigualdades no acesso à saúde impactam diretamente o diagnóstico, o tratamento e o prognóstico de diversas doenças, dentre elas as da tireoide ${ }^{1,3,5,12,14,15}$. Estudos relatam diferenças nos fatores 
Tabela 2. Comparação entre as características clínico-patológicas dos pacientes com neoplasias malignas

\begin{tabular}{|c|c|c|c|}
\hline \multirow{3}{*}{$\begin{array}{l}\text { Características clínico-patológicas } \\
\text { dos pacientes com doença maligna }\end{array}$} & $\begin{array}{l}\text { SUS } \\
\mathrm{n}=34\end{array}$ & $\begin{array}{c}\text { Saúde } \\
\text { Suplementar } \\
\mathrm{n}=65\end{array}$ & \multirow{3}{*}{ p } \\
\hline & \multicolumn{2}{|c|}{ Total $(n=99)$} & \\
\hline & \multicolumn{2}{|c|}{$n(\%)$} & \\
\hline $\begin{array}{l}\text { Tipo de tumor } \\
\text { Carcinoma Papilifero } \\
\text { Carcinoma Folicular } \\
\text { Carcinoma Anaplásico } \\
\text { Carcinoma Medular } \\
\text { Carcinoma de Células de Hurthle }\end{array}$ & $\begin{array}{c}29(85,3) \\
3(8,9) \\
- \\
1(2,9) \\
1(2,9) \\
\end{array}$ & $\begin{array}{c}59(90,8) \\
2(3,1) \\
1(1,5) \\
- \\
3(4,6) \\
\end{array}$ & 0,411 \\
\hline $\begin{array}{l}\text { Estadiamento Patológico T } \\
1 \\
1 \mathrm{a} \\
1 \mathrm{~b} \\
1 \mathrm{c} \\
2 \\
3 \\
3 \mathrm{a} \\
4 \\
4 \mathrm{a} \\
4 \mathrm{~b} \\
\end{array}$ & $\begin{array}{c}13(38,2) \\
- \\
- \\
1(1,0) \\
14(41,2) \\
1(1,0) \\
- \\
4(11,8) \\
1(1,0) \\
- \\
\end{array}$ & $\begin{array}{c}28(43,2) \\
2(3,1) \\
3(4,6) \\
- \\
22(33,8) \\
7(10,8) \\
1(1,5) \\
1(1,5) \\
- \\
1(1,5 \%)\end{array}$ & 0,259 \\
\hline $\begin{array}{l}\text { Estadiamento Patológico } \mathrm{N} \\
0 \\
1 \\
1 \mathrm{a} \\
1 \mathrm{~b} \\
\mathrm{x}\end{array}$ & $\begin{array}{l}17(50,0) \\
5(14,8) \\
2(5,8) \\
4(11,8) \\
6(17,6)\end{array}$ & $\begin{array}{c}26(40,0) \\
9(13,8) \\
4(6,2) \\
8(12,3) \\
18(27,7) \\
\end{array}$ & 0,339 \\
\hline $\begin{array}{l}\text { Estadiamento Patológico M } \\
0 \\
1 \\
\mathrm{x} \\
\end{array}$ & $\begin{array}{c}8(23,6) \\
1(2,9) \\
25(73,5) \\
\end{array}$ & $\begin{array}{c}5(7,7) \\
1(1,5) \\
59(90,8) \\
\end{array}$ & 0,030 \\
\hline $\begin{array}{l}\text { Estadiamento Clínico } \\
\text { I } \\
\text { II } \\
\text { III } \\
\text { IVa } \\
\text { IVb }\end{array}$ & $\begin{array}{c}23(67,7) \\
5(14,7) \\
3(8,8) \\
2(5,9) \\
1(2,9) \\
\end{array}$ & $\begin{array}{l}49(75,4) \\
4(6,2) \\
4(6,2) \\
7(10,7) \\
1(1,5) \\
\end{array}$ & 0,767 \\
\hline
\end{tabular}

Estadiamento patológico: T: tumor primário; N: comprometimento de linfonodos; M: metástases regionais.

de risco e na apresentação clínica das neoplasias tireoidianas, como a gravidade da doença e sobrevida, relacionadas a fatores socioeconômicos e de acesso à saúde $\mathrm{e}^{12-16}$.

O presente estudo teve como objetivo verificar a influência do tipo de acesso à saúde na apresentação clínica e histopatológica das neoplasias tireoidianas benignas e malignas no momento do tratamento em pacientes submetidos à tireoidectomia total na Cidade de Guarapuava, Paraná, Brasil. A cidade é sede de uma regional de saúde do estado, que abrange mais dezenove municípios vizinhos e tem uma estimativa de $3,14 \%$ da população considerada como extrema pobreza e 14,56\% têm acesso a planos de saúde privados ${ }^{23}$.

A literatura evidencia que indivíduos socioeconomicamente desfavorecidos possuem um acesso limitado à saúde, têm maior dificuldade em conseguir serviços especializados e com frequência residem em áreas pobres e/ou afastadas de centros 
Tabela 3. Fatores demográficos e clínicos associados ao volume da glândula estimado pela ultrassonografia

\begin{tabular}{|c|c|c|}
\hline \multirow{2}{*}{ Variáveis } & Volume da glândula tireoide & \multirow[b]{2}{*}{$\mathrm{p}$} \\
\hline & Mediana (P25 - P75) & \\
\hline $\begin{array}{l}\text { Idade } \\
<45 \text { Anos } \\
245 \text { Anos } \\
\end{array}$ & $\begin{array}{c}22,40(14,30-41,80)^{*} \\
35,78(18,90-72,70)\end{array}$ & $<0,001$ \\
\hline $\begin{array}{l}\text { Sexo } \\
\text { Feminino } \\
\text { Masculino }\end{array}$ & $\begin{array}{c}27,85(14,80-53,68)^{*} \\
46,34(21,20-81,10)\end{array}$ & $<0,001$ \\
\hline $\begin{array}{l}\text { Plano de saúde } \\
\text { SUS } \\
\text { Saúde Suplementar }\end{array}$ & $\begin{array}{l}46,61(21,53-115,44) \\
24,70(14,63-46,80)^{*}\end{array}$ & $<0,001$ \\
\hline $\begin{array}{l}\text { Presença concomitante de doença tireoidiana } \\
\text { Nenhuma } \\
\text { Doença de Graves } \\
\text { Tireoidite de Hashimoto } \\
\text { Tireoidite Linfocítica }\end{array}$ & $\begin{array}{c}30,05(16,80-61,66) \\
60,99(-) \\
14,70(11,30-35,10)^{*} \\
23,80(15,70-46,41)\end{array}$ & $<0,001$ \\
\hline $\begin{array}{l}\text { Diagnóstico patológico } \\
\text { Benigno } \\
\text { Maligno }\end{array}$ & $\begin{array}{c}32,75(18,55-69,78) \\
21,96(12,40-46,34)^{*}\end{array}$ & $<0,001$ \\
\hline
\end{tabular}

Volume da glândula tireoide: $\mathrm{cm}^{3}$. "menor significativamente que os demais grupos.

Tabela 4. Fatores demográficos e clínicos associados ao peso do espécime cirúrgico

\begin{tabular}{|c|c|c|}
\hline \multirow[b]{2}{*}{ Variáveis } & Peso do espécime cirúrgico & \multirow[b]{2}{*}{ p } \\
\hline & MD (P25 - P75) & \\
\hline $\begin{array}{l}\text { Idade } \\
<45 \text { Anos } \\
245 \text { Anos } \\
\end{array}$ & $\begin{array}{c}25(15-40)^{*} \\
45(22-90)\end{array}$ & $<0,001$ \\
\hline $\begin{array}{l}\text { Sexo } \\
\text { Feminino } \\
\text { Masculino } \\
\end{array}$ & $\begin{array}{c}30(20-67) \\
37,5(25-84) \\
\end{array}$ & 0,105 \\
\hline $\begin{array}{l}\text { Plano de saúde } \\
\text { SUS } \\
\text { Saúde Suplementar }\end{array}$ & $\begin{array}{c}40(25-130) \\
25,5(15-60)^{*}\end{array}$ & $<0,001$ \\
\hline $\begin{array}{l}\text { Presença concomitante de doença tireoidiana } \\
\text { Nenhuma } \\
\text { Doença de Graves } \\
\text { Tireoidite de Hashimoto } \\
\text { Tireoidite Linfocítica }\end{array}$ & $\begin{array}{c}30(20-75) \\
67(--) \\
20(15-25)^{*} \\
30(25-45)\end{array}$ & $<0,001$ \\
\hline $\begin{array}{l}\text { Diagnóstico patológico } \\
\text { Benigno } \\
\text { Maligno }\end{array}$ & $\begin{array}{l}40(20-87,5) \\
25(15-35)^{*}\end{array}$ & $<0,049$ \\
\hline
\end{tabular}

Peso: g. "menor significativamente que os demais grupos. 
urbanos, o que dificulta a prevenção secundária, resultando em diagnósticos em estágios avançados 1,14,15,24. Além disso, esses também são fatores que interferem na adesão ao tratamento, visto que o alto custo de medicamentos ou procedimentos pode ser uma barreira para aqueles pacientes com recursos financeiros limitados ${ }^{15}$.

Os resultados obtidos em nosso estudo confirmam tais evidências, pois, mostraram que existe uma diferença significativa entre os pacientes atendidos pelo SUS e pela saúde suplementar quanto ao volume total da tireoide, peso do espécime cirúrgico e metástases regionais, além de associações entre as características demográficas e clínicas. Pacientes atendidos pelo SUS podem ter enfrentado maior dificuldade no acesso ao diagnóstico da doença e maior tempo de espera para a realização da cirurgia, fatores que podem ter implicado nestes achados.

Wu et al. ${ }^{1}$ encontraram resultados semelhantes ao nosso estudo. Os autores buscaram avaliar a influência das condições socioeconômicas em pacientes que realizaram tireoidectomia total, comparando os que possuíam seguros privados de saúde e aqueles que não possuíam ou que participavam de algum programa público de saúde. Seus resultados, a partir da análise de 252 prontuários, mostraram uma associação significativa entre o estado socioeconômico com o seguro de saúde $(p<0,001)$, raça $(p<0,001)$ e volume total da tireoide $(p=0,016)$. Nos tumores benignos o fator socioeconômico foi o preditor de maior significância para volumes tireoidianos maiores, enquanto nos tumores malignos, o grupo sem seguro ou com seguro de saúde pública foi associado a um maior número de nódulos positivos $(p=0,002)$, margens positivas $(p=0,008)$ e maior número de metástases $(p=0,023)$. Em nosso estudo também identificamos diferenças significativa entre os grupos na presença de metástases regionais ( $p=0,03)$, assim como no estudo de Harari et al. ${ }^{15}$, no qual os autores identificaram maior presença de metástases em pacientes sem seguros de saúde ou com seguros de saúde públicos (OR =2,41; IC:2,10-2,77).

Nossa amostra apresentou homogeneidade entre os grupos em relação à idade, porém, foi observado que pacientes acima de 45 anos apresentaram tireoides com maior volume estimado pela US $\left(35,78 \mathrm{~cm}^{3}\right.$ versus $22,40 \mathrm{~cm}^{3} ; p<0,001$ ) e maior peso do espécime cirúrgico ( $45 g$ versus $25 g$; $p<0,001$ ). A literatura observa dados semelhantes em relação às neoplasias malignas de tireoide, Zevallos et al. ${ }^{14}$, ao avaliarem a influência socioeconômica no tratamento de pacientes com câncer papilífero, identificaram que pacientes com idade igual ou superior a 45 anos considerados como baixo status socioeconômico apresentaram tumores com volume maior $(p=0,002)$ e com extensão extratireoidiana $(p=0,035)$, tumores em estágio clínico mais avançado $(p<0,001)$, multifocais $(p=0,013)$ e com linfonodos positivos $(p=0,018)$.

Nossos resultados mostram uma associação entre o volume tireoidiano e o tipo de seguro saúde, sendo $46,61 \mathrm{~cm}^{3}$ a mediana entre os casos atendidos pelo SUS e $24,70 \mathrm{~cm}^{3}$ os casos operados via saúde suplementar $(p<0,001)$. Tireoides de grande volume, de acordo com literatura, 
estão associadas à presença mais frequente de neoplasia maligna ${ }^{25}$. O estudo de Lima et al. ${ }^{25}$ investigou a prevalência de nódulos tireoidianos e preditores de malignidade em pacientes com doenças autoimunes de tireoide, identificando que grandes volumes tireoidianos estavam associados à presença de neoplasias malignas (OR $=1.018 ; 95 \%$ IC $=1.005-1.030 ; p=0.005)$.

Erbil et al. ${ }^{26}$ relataram associação do volume tireoidiano e do tamanho do tumor com a detecção de nódulos com suspeita de malignidade (OR: 48; $p<0,001$ e OR: 21,5; $p<0,001)$. Os autores observaram que quanto menor o volume melhor a eficácia do exame para a detecção, enquanto, em tireoides volumosas, o exame de US pode perder a sensibilidade na detecção de tais nódulos, retardando e dificultando o tratamento. Zagzag et al. ${ }^{27}$, ao compararem a incidência de detecção de câncer de tireoide em um hospital público e um privado, observaram que além dos pacientes do hospital público possuírem tumores maiores $(23 \mathrm{~mm}$ versus $18 \mathrm{~mm} ; \mathrm{p}=0,04)$ no momento do diagnóstico, os pacientes do hospital privado possuíam mais chances de ter os tumores detectados pela US ( $46 \%$ versus $28 \%$; $p<0,01)$.

Em estudo retrospectivo, Banks et al. ${ }^{28}$ identificaram uma associação entre grandes volumes tireoidianos e aumento dos sintomas compressivos. O estudo analisou 333 casos de pacientes submetidos à tireoidectomia pelo Serviço de Cirurgia de Cabeça e Pescoço da Universidade Médica da Carolina do Norte nos EUA, observando que $52 \%$ dos pacientes apresentavam sintomas compressivos incluindo disfagia e dispneia, sendo que a média do volume da tireoide dos pacientes com sintomas compressivos foi maior do que aqueles que não apresentaram tais sintomas $\left(75,5 \mathrm{~cm}^{3}\right.$ versus $\left.37,1 \mathrm{~cm}^{3} ; p<0,001\right)$.

A cirurgia da tireoide apresenta técnica bem estabelecida e segura, entretanto, um volume tireoidiano aumentado além de tornar a cirurgia mais longa e complexa, pode causar a compressão e desvio da traqueia ocasionando sintomas importantes no préoperatório, além de aumentar a morbidade pós-operatória implicando em maior tempo de internação e elevação de custos. Existe ainda um risco maior de lesão do nervo laríngeo recorrente $(\mathrm{NLR})^{29,30}$. Testini et al. ${ }^{29}$ ao verificarem os fatores que poderiam aumentar o risco de lesões no NLR em uma tireoidectomia total, observaram que tireoides com volumes maiores apresentavam um aumento no risco de lesão do NLR.

Lesões no NLR determinam imobilidade das pregas vocais. Nas tireoidectomias esse tipo de complicação ocorre de 0 a $7,1 \%$ nas formas temporárias e de 0 a $11 \%$ nas definitivas. Esse índice eleva-se nos casos de reoperações até $20 \%$, como nas recidivas linfonodais do compartimento central ${ }^{31}$. Durante as tireoidectomias é fundamental a visualização e dissecção do NLR, bem como, o exame das pregas vocais é importante na avaliação pré e pós-operatória das tireoidectomias ${ }^{31-33}$.Para auxiliar na prevenção de lesões do NLR, a MION (monitorização intraoperatória do nervo), permite avaliar a função neural e facilita a identificação dos nervos e seus ramos, sendo principalmente útil em tumores volumosos $^{31,34}$. A MION está indicada nas 
cirurgias de maior risco, especialmente em situações como reoperações, tumores com invasão extra capsular, tumores volumosos e em área previamente irradiada ${ }^{31}$. No entanto, esse recurso aumenta os custos da cirurgia e em muitos casos, na maioria dos serviços não é custeada pelos serviços públicos de saúde.

Em relação aos pacientes do nosso estudo, apesar de não termos avaliado os índices de complicações cirúrgicas, tais como lesões do NLR, o maior volume tireoidiano identificado nos pacientes atendidos pelo SUS é um fator de risco para complicações, dentre elas a lesão do NLR. Embora este estudo não tenha analisado o índice de complicações pósoperatórias e evolução clínica dos pacientes, nossos resultados, ao comparar pacientes atendidos pelo SUS e por saúde suplementar, evidenciaram o impacto das desigualdades em saúde na apresentação clínica das neoplasias tireoidianas.

Aproximadamente $75 \%$ da população brasileira depende exclusivamente do SUS ${ }^{35}$. Em virtude de baixo investimento e má gestão, o sistema único enfrenta a escassez de serviços disponíveis, e dessa forma os pacientes sofrem com os vieses de diagnóstico tardio e as consequentes morbimortalidades associadas a este es, $^{356}$.

\section{CONCLUSÃO}

Pacientes com baixas condições socioeconômicas e que dependem totalmente do SUS são afetados pelas dificuldades que permeiam esse sistema. $\mathrm{Na}$ amostra deste estudo, a realização do tratamento pelo SUS determinou um volume total da tireoide e peso do espécime cirúrgico maiores, bem como maior ocorrência de metástases regionais, demonstrando que a desigualdade no acesso à saúde influencia no diagnóstico e apresentação da doença.

\section{REFERÊNCIAS}

1. Wu TJ, Ha PK, El-Sayed IH, George JR, Heaton CM, Ryan WR, et al. Impact of socioeconomic status on presentation among urban patients undergoing total thyroidectomy. The Laryngoscope. 2016;126(12):1-28.

2. Barreto ML. Health inequalities: a global perspective. Ciênc. Saúde Coletiva. 2017;22(7):2097-108.

3. Barros MBA. Desigualdade social em saúde: revisitando momentos e tendências nos 50 anos de publicação da RSP. Rev Saúde Pública. 2017;51(17):1-8.

4. Barata, RB. O que queremos dizer com desigualdades sociais em saúde? In: Como e por que as desigualdades sociais fazem mal à saúde. Rio de Janeiro, RJ: Editora Fiocruz. Coleção Temas em Saúde; 2009:11-21.

5. Ribeiro AA, Nardocci AC. Socioeconomic inequalities in cancer incidence and mortality: review of ecological studies, 1998-2008. Saúde Soc. 2013;22(3):878-891.

6. Wunsch Filho V, Antunes JLF, Boing AF, Lorenzi RL. Prospects of research on social determinants in cancer. Physis: Revista de Saúde Coletiva. 2008;18(3):427-450.

7. Keane E, Francis EC, Catháin EO, Rowley H. The role of race in thyroid cancer: systematic review. J Laryngol Otol. 2017;131(6):480-486.

8. Vander JB, Gaston EA, Dawber TR. The significance of nontoxic thyroid nodules. Final report of a 15 -year study of the incidence of thyroid malignancy. Ann Intern Med $1968 ; 69: 537-540$. 
9. Brander AE, Viikinkoski VP, Nickels JI, Kivisaari LM. Importance of thyroid abnormalities detected at US screening: a 5- year follow-up. Radiology 2000; 215:801806.

10. Matos PS, Ferreira AP, Ward LS. Prevalence of papillary microcarcinoma of the thyroid in Brazilian autopsy and surgical series. Endocr Pathol. 2006; 17:165-173.

11. Hayat MJ, Howlader N, Reichman ME, Edwards BK. Cancer statistics, trends, and multiple primary cancer analyses from the Surveillance, Epidemiology, and End Results (SEER) program. Oncologist 2007;12:20-37.

12. ICCIDD - International Council for the Control of lodine Deficiency Disorders. ICCIDD global network.

13. Mesele M, Degu G, Gebrehiwot H. Prevalence and associated factors of goiter among rural children aged 6-12 years old in Northwest Ethiopia, cross-sectional study. BMC Public Health.2014;14:130.

14. Zevallos JP, Xu L, Yiu Y. The impact of socioeconomic status on the use of adjuvant radioactive iodine for papillary thyroid cancer. Thyroid. 2014; 24(4):758-763.

15. Harari A, Li N, Yeh MW. Racial and socioeconomic disparities in presentation and outcomes of welldifferentiated thyroid cancer. J Clin Endocrinol Metab. 2014; 99(1):133-141.

16. Lim, II, Hochman T, Blumberg SN, Patel KN, Heller $\mathrm{KS}$, Ogilvie JB. Disparities in the initial presentation of differentiated thyroid cancer in a large public hospital and adjoining university teaching hospital. Thyroid. 2012; 22(3):269-274.

17. IBGE [https://cidades.ibge.gov.br/brasil/pr/guarapuava/ panorama]. Coordenação de População e Indicadores Sociais: Instituto Brasileiro de Geografia e Estatística; 2019. Atualizado $1^{\circ}$ de julho de 2019.

18. Hochman B, Nahas FX, Oliveira Filho RS, Ferreira LM. Research designs. Acta Cir. Bras. 2005; 20(2):1-9.

19. Knudsen N, Bols B, Bulow I, Jørgensen $T$, Perrild $H$, Ovesen L, et al. Validation of ultrasonography of the thyroid gland for epidemiological purposes. Thyroid. 1999; 9(11):1069-1074.

20. Brauer VF, Eder P, Miehle K, Wiesner TD, Hasenclever $H$, Paschke R. Interobserver variation for ultrasound determination of thyroid nodule volumes. Thyroid. 2005;15(10):1169-1175.

21. Kaniuka-Jakubowska S, Piskunowicz M, Zapasnik A, Lewczuk A, Kaniuka A, Mizan-Gross K, et al. US not bright but right method of thyroid volume estimation even in large and substernal extended goitres. Comparison of US and three methods of CT thyroid evaluation - prospective study. Clin Endocrinol (Oxf). 2015; 83(3):412-419.

22. American Joint Committee on Cancer (AJCC) TNM Staging for thyroid-Differentiated and Anaplastic Carcinoma $\left(8^{\text {th }}\right.$ ed., 2017).

23. SESA. $5^{\text {a }}$ Regional de Saúde: Secretaria de Saúde; 2019. Atualizado dezembro de 2018.

24. Keegan TH, Grogan RH, Parsons HM, Tao L, White MG, One K, et al. Sociodemographic disparities in differentiated thyroid cancer survival among adolescents and young adults in California. Thyroid, 2015; 25(6):635-648.

25. Lima PC, Moura Neto A, Tambascia MA, Zantut Wittmann $D E$. Risk factors associated with benign and malignant thyroid nodules in autoimmune thyroid diseases. ISRN Endocrinol. 2013; 2013:1-7.

26. Erbil $Y$, Barbaros U, Salmaslioglu A, Mete O, Issever $\mathrm{H}$, Ozarmagan S, et al. Effect of thyroid gland volume in preoperative detection of suspected malignant thyroid nodules in a multinodular goiter. Arch Surg. 2008;143(6):5585-63.

27. Zagzag J, Kenigsberg A, Patel KN, Heller KS, Ogilvie JB. Thyroid cancer is more likely to be detected incidentally on imaging in private hospital patients. J Surg Res. 2017; 1(6):239-244

28. Banks CA, Ayers CM, Hornig JD, Lentsch EJ, Day TA, Nguyen SA, et al. Thyroid disease and compressive symptoms. Laryngoscope. 2012; 122(1):13-16.

29. Testini M, Gurrado A, Bellantone R, Brazzarola P, Cortese $\mathrm{R}$, De Toma G, et al. Recurrent laryngeal nerve palsy and substernal goiter. An Italian multicenter study. J Visc Surg. 2014;151(3):183-189.

30. Abboud B, Sleilaty G, Mallak N, Abou Zeid H, Tabchy B. Morbidity and mortality of thyroidectomy for substernal goiter. Head Neck. 2010; 32(6):744-749.

31. Kim MK, Mandel SH, Baloch Z, Livolsi VA, Langer JE, Didonato $\mathrm{L}$, et al. Morbidity following central compartment reoperation for recurrent or persistent thyroid cancer. Arch Otolaryngol Head Neck Surg. 2004;130(10):1214-1216.

32. Lahey FH. Routine dissection and demonstration of the recurrent laryngeal nerve in subtotal thyroidectomy. Surg Gynecol Obstet. 1938; 66:775-777.

33. Riddell VH. Injury to recurrent laryngeal nerves during thyroidectomy - a comparison between the results of identification and non-identification in 1022 nerves exposed to risk. Lancet.1956; 29:638-461. 
34. Shindo M, Chheda NN. Incidence of vocal cord paralysis with and without recurrent laryngeal nerve monitoring during thyroidectomy. Arch Otolaryngol Head Neck Surg. 2007; 133(1):481-485.

35. Celuppi IC, Geremia DS, Ferreira J, Pereira AMM, Souza JB. 30 anos de SUS: relação público-privada e os impasses para o direito universal à saúde. Saúde Debate. 2019; 43(121):302-313.
36. Fahel M, Neves J. Desigualdades em Saúde no Brasil: uma análise do acesso aos serviços de saúde por estratos ocupacionais. Ver Teor e Soc. 2007; 17(2):140-169.

\section{CORRESPONDÊNCIA}

Suzane Cristina Santos

Rua Xavier da Silva 1194 apto 06, Centro

Guarapuava, PR CEP: $85010-220$

Email: suzanecristinaa@gmail.com 